\title{
Multiple Degree of Freedom Model of Giant Magnetostrictive Actuator
}

\author{
Dongwei LI ${ }^{1,2}{ }^{*}$, Zhongbo HE ${ }^{1}$, Peilin ZHANG ${ }^{1}$, Zhaoshu YANG ${ }^{1}$, Ce RONG ${ }^{1}$, Yezun SUN ${ }^{3}$ \\ ${ }^{1}$ Vehicles and Electrical Engineering Department, Ordnance Engineering College, Heping West 97, Shijiazhuang 050003, \\ China \\ ${ }^{2}$ College of Mechanism and Vehicle, Beijing Institute of Technology, Beijing 100081 China \\ ${ }^{3} 247$ Military Representative Office, GAD Military Representative Bureau, Taiyuan 030009, China
}

cross ref http://dx.doi.org/10.5755/j01.ms.23.3.16321

Received 30 September 2016; accepted 25 December 2016

\begin{abstract}
A specific giant magnetostrictive actuator (GMA) is developed for injectors in high-pressure-common-rail system. The magnetic strength, with its axial distribution unevenness considered, is determined by the driving current and axial dimension. Multiple degrees of freedom model of the specific GMA is established based on Jiles-Atherton (J-A) model and vibration theory. The number of degrees of freedom is optimized, and step response is calculated and its state-space model is provided and computed using MATLAB. Steady-state response and frequency response in moderate driving level is achieved and verified by the test results, and load contributed by fluid is regarded as elastic-damping load and the specific response of GMA in this case could be forecast. From the measured result, the proposed multiple degrees of freedom model are amenable to describe the stable response of the specific GMA under low frequency harmonic driving. Keywords: giant magnetostrictive actuator, multiple degrees of freedom model, stable response, harmonic response, oil load.
\end{abstract}

\section{INTRODUCTION}

Giant magnetostrictive material (GMM) enjoys plenty of merits stretches from fast response, bulky output force to high energy transfer efficiency, the development and application of GMM is therefore becomes a thriving scope for researchers. Engineering application is an important aspect for the giant magnetostrictive material and there are multiple structures in Giant magnetostrictive actuator (GMA) design, E. Quandt [1] developed a cantilever actuator based on GMM films to provide precise fluid control under low driving level. F. Claeyssen [2] weighed the characteristics of different actuators, transducers and motors based on GMM and provided a roadmap to select an appropriate structure in GMA design. Young-Woo Park [3] developed a micro GMA structure which could provide a precise displacement control. Jiaju Zheng [4] developed a magnetostrictive force controller which is free from coil and therefore avoids the Joule heat. Yuesong Li [5] employed DC bias field and heat compensator into GMA structure and the field unevenness was studied. Bintang Yang [6] combined the GMA with and without permanent magnets, and with the help of a mechanical amplifier, the stroke of GMA reached $0.71 \mathrm{~mm}$.

In this paper, a specific GMA is developed to drive the globe valve within an injector serving in high-pressurecommon-rail system. The objective of this work concerns developing a GMA based injector, which can operate properly [7] as well as maintain a swift response under a driving voltage of $24 \mathrm{~V}$, which is the ceil provided by vehicle batteries [8]. With respect to the GMA serving in the electric injectors, a Sweden company [9] developed a GMM injector without mechanical connection to realize a

\footnotetext{
${ }^{*}$ Corresponding author. Tel.: +086-0311-87994743.

E-mail address: $295159081 @ q q . c o m$ (D.W. Li)
}

non-resolution control of fuel injection; Tanaka [10] designed a Z-type GMM driver serving in oil injector to achieve a $40 \mu \mathrm{m}$ stroke output; Fuzai Lv [11] developed a GMM switching valve which could provide both considerable stroke and output force. All the above structures show remarkable merits but fail to fit directly into the injector case, since their working principle are based on the elongation of GMM rod while the normally closed injector requires the actuator to shrink after excitement. This issue is resolved in this work by a series of structure design and modification.

A single degree of freedom (DOF) model for GMA regard the GMM rod as a single stiffness-mass-damping device, or computing the displacement by multiplying magnetostriction directly with rod length. These methods are proposed based on the assumption that the axial distribution of magnetic strength is uniform and the strain distribution is even. However, when the axial dimension is limited, the uniform of magnetic field cannot be ensured and correctness of this single DOF model is questionable [12]. As a type of engineering applied material, a precise model for the giant magnetostrictive actuator is quite urgent.

\section{OPERATING PRINCIPLE}

The proposed GMA structure is detailed as Fig. 1, the adjustment bolt is connected by screw with one end of displacement output rod, and the alternative end is connected with a test rod. Therefore, the screw cap, output rod and test rod could be regarded as a single part in dynamic analysis.

With respect to the thick end of output shaft, a rectangular groove is manufactured to facilitate the relative displacement of slider and output shaft. Since there is no bias field exerted, when excited, the slider will fix the 
lower end of GMM bar and force the alternative end to move ahead against the spring force. In this way, a corresponding force and displacement is generated.

According to the local zoom in Fig. 1, there is a gap between the output rod and slider, the length of this gap should be kept larger than the maximum elongation of GMM bar. Another gap should be provided between output shaft and shell of GMA to protect the shaft from colliding to shell when the power is off.

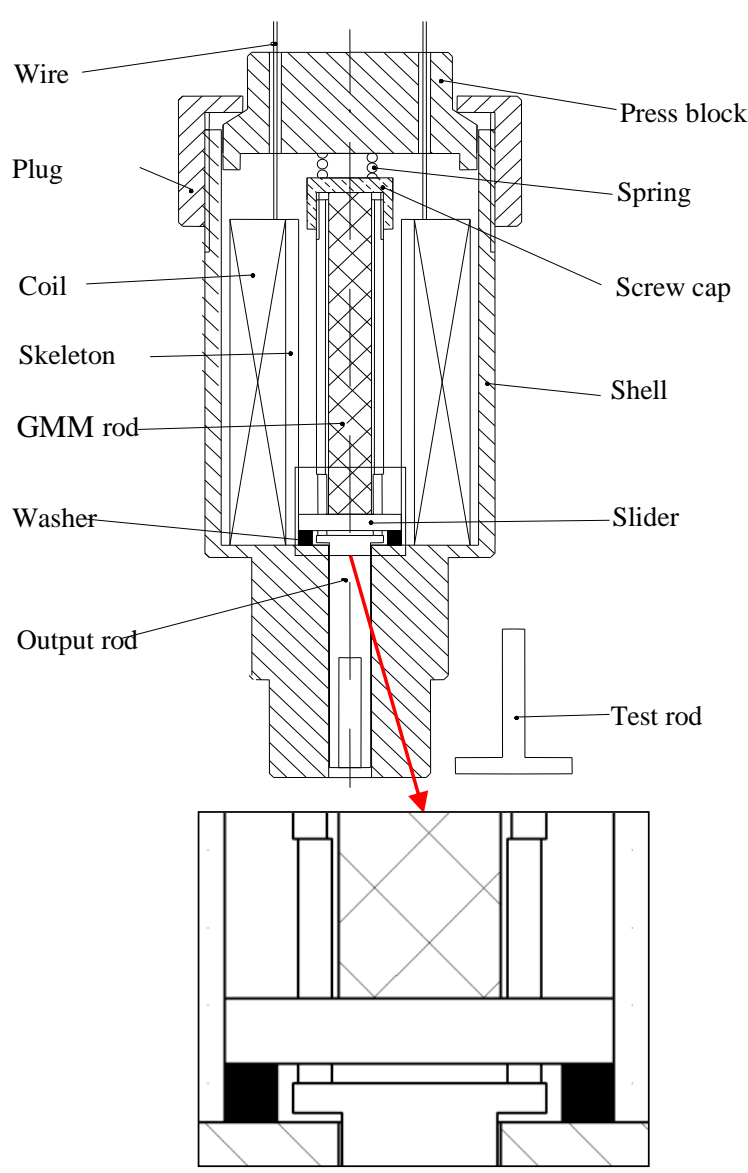

Fig. 1. Structure and local zoom of GMA for oil injector

\section{MODEL OF GMA AND RESPONSE SOLUTION}

According to previous analysis, a multi-coupled model of the specific GMA is established including fields of current, magnetic field and mechanics [13].

\subsection{Magnetostriction model}

In GMA structure, a multilayer coil is employed, its profile is exhibited as Fig. 2. Since the aspect ratio of coil section is not large enough and the GMM rod is not located solely within the strong field domain of driving coil, the axial field distribution is uneven. According to Fig. 1, the magnetic circuit of GMA is not closed. In this case, the classical magnetic circuit method cannot be applied directly. According to Ampere's Law, the magnetic field of a solenoid with unlimited length is integrated. The coil is simplified as Fig. 2. Under DC or low frequency driving current $I$, a single layer coil with thickness dr will generate a axial magnetic field in point $(z, 0)$.

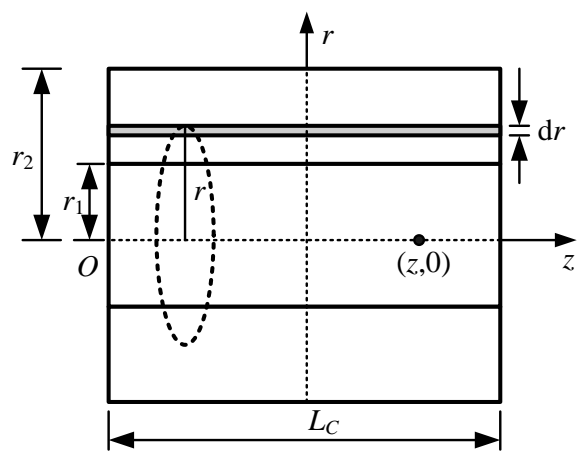

Fig. 2. Profile of a multilayer coil

The magnetic field intensity could be calculated via:

$$
\mathrm{d} H=\frac{N I \mathrm{~d} r}{2 L_{C}\left(r_{2}-r_{1}\right)}\left(\frac{\frac{L_{C}}{2}-z}{\sqrt{\left(\frac{L_{C}}{2}-z\right)^{2}+r^{2}}}+\frac{\frac{L_{C}}{2}+z}{\sqrt{\left(\frac{L_{C}}{2}+z\right)^{2}+r^{2}}}\right)(1)
$$

where $N$ denotes the turns of the coil, $L_{\mathrm{C}}, r_{1}$ and $r_{2}$ denote respectively the length, inner and outer radii of coil, $N I /\left[L_{C} *\left(r_{2}-r_{1}\right)\right]$ denotes the surface current density of coil. The total field intensity along the axis can be calculated as the integration of Eq. (1) within the region of $\left[r_{1}, r_{2}\right]$.

$$
\begin{aligned}
& H(I, z)=\frac{N I}{2 L_{C}\left(r_{2}-r_{1}\right)}\left[\left(\frac{L_{C}}{2}-z\right)\right. \\
& \left.\ln \frac{r_{2}+\sqrt{\left(\frac{L_{C}}{2}-z\right)^{2}+r_{2}^{2}}}{r_{1}+\sqrt{\left(\frac{L_{C}}{2}-z\right)^{2}+r_{1}^{2}}}+z \ln \frac{r_{2}+\sqrt{\left(\frac{L_{C}}{2}-z\right)^{2}+r_{2}^{2}}}{r_{1}+\sqrt{\left(\frac{L_{C}}{2}-z\right)^{2}+r_{1}^{2}}}\right]
\end{aligned}
$$

Jiles-Atherton ( $\mathrm{J}-\mathrm{A})$ model is commonly used to describe the hysteresis behavior of GMM devices, which is based on domain wall theory [14-16]. There are five constituting equations in $\mathrm{J}$-A model which reveals the relationship between exciting field intensity $H$, effective field intensity $H_{\mathrm{e}}$, anhysteresis magnetization $M_{\text {an }}$, irreversible magnetization $M_{\text {irr }}$, reversible magnetization $M_{\text {rev }}$ as well as the total magnetization $M$.

$\left\{\begin{array}{l}H_{\mathrm{e}}=H+\tilde{\alpha} M \\ M_{\mathrm{an}}=M_{\mathrm{s}}\left[\operatorname{coth}\left(H_{\mathrm{e}} / a\right)-a / H_{\mathrm{e}}\right] \\ \frac{\mathrm{d} M_{\mathrm{irr}}}{\mathrm{d} H}=\frac{M_{\mathrm{an}}-M_{\mathrm{irr}}}{\delta k-\tilde{\alpha}\left(M_{\mathrm{an}}-M_{\mathrm{irr}}\right)} \\ M_{\mathrm{rev}}=c\left(M_{\mathrm{an}}-M_{\mathrm{irr}}\right) \\ M=M_{\mathrm{irr}}+M_{\mathrm{rev}}\end{array}\right.$.

Above equation could be deduced as Eq. 4 


$$
\left\{\begin{array}{rl}
\frac{\mathrm{d} M}{\mathrm{~d} H}= & \frac{M_{\mathrm{s}} c\left(1 / z^{2}+1-\operatorname{coth}^{2} z\right)}{a-M_{\mathrm{s}} c \tilde{\alpha}\left(1 / z^{2}+1-\operatorname{coth}^{2} z\right)}+ \\
& \left\{\frac{a\left[M_{s}(\operatorname{coth} z-1 / z)-M\right]}{\delta k-\tilde{\alpha}\left[M_{s}(\operatorname{coth} z-1 / z)-M\right] /(1-c)}\right\}^{a-M_{\mathrm{s}} c \tilde{\alpha}\left(1 / z^{2}+1-\operatorname{coth}^{2} z\right)}
\end{array},\right.
$$

where $z=(H+\tilde{\alpha} M) / a$. In Eq. $4, \alpha$ is the magnetic field parameter, $c$ is domain wall flexing parameter, $a$ is the shape parameter, $k$ is domain wall pinning parameter, $M_{\mathrm{s}}$ denotes saturated magnetization, $\delta$ is a symbolic parameter and $\delta=+1$ when $H$ increases while $\delta=-1$ when $H$ decreases.

The relationship between axial magnetostriction and magnetization follows Eq. 5, where $\lambda_{\mathrm{s}}$ denotes the saturated magnetostriction.

$$
\lambda(I, z)=\frac{3 \lambda_{\mathrm{s}}}{2 M_{\mathrm{s}}^{2}} M^{2} .
$$

\subsection{Multi-DOFs model}

According to Eq. 2, Eq. 4 and Eq. 5, the magnetostriction distribution of GMM bar is uneven along axis. Correspondingly, the driving force along the axis is also uneven. In this case, a single DOF spring-dampingmass system usually fails to count for this complicated system since the force distribution in a single DOF system is assumed uniformly distributed. In this paper, considering the specific axial distribution of magnetostriction, GMM rod is regarded as a multi-DOF system, and its corresponding model is detailed as Fig. 3.

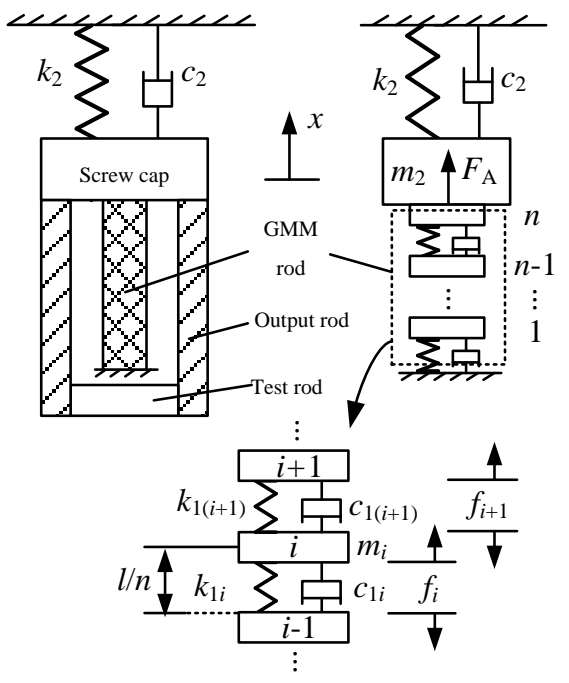

Fig. 3. Equivalent multi-DOFs model of GMA

A GMM rod is divided into $n$ elements with equal length. Each element is equivalent as a spring-dampingmass system, and the kinetic energy of $i^{\text {th }}$ element, which is counted through its bottom, is assumed to be lumped to the equivalent mass.

$$
\frac{1}{2} m_{i}\left(\frac{i}{n} v\right)^{2}=\int_{\frac{i-1}{n} L}^{\frac{i}{n} L} \frac{1}{2} \rho A\left(\frac{x}{L} v\right)^{2} \mathrm{~d} x,
$$

where $A, L$ and $\rho$ denotes respectively the section area, length and density of GMM rod, $v$ denotes the velocity of its free end.

Therefore, the equivalent mass for ith element is calculated as

$m_{i}=\frac{\rho A L}{n}\left(1-\frac{1}{i}+\frac{1}{3 i^{2}}\right)$.

The equivalent stiffness and damping ratio for $i$ th element are

$\left\{\begin{array}{l}k_{i}=k_{\mathrm{r}}=A E^{H} /(L / n) \\ c_{i}=c_{\mathrm{r}}=A C_{\mathrm{D}} /(L / n)\end{array} \quad i=1,2, \ldots, n\right.$,

where $E^{H}$ is elastic module of GMM bar, $C_{\mathrm{D}}$ is damping of GMM rod, $m_{\mathrm{f}}$ is total equivalent mass of bolt, output shaft and test bar, $k_{\mathrm{f}}$ and $c_{\mathrm{f}}$ are the equivalent stiffness of disk spring and damping coefficient. Combining the load with the $n$th mass and taking the equilibrium point as origin, the multi-DOF dynamic model for the specific actuator could be setup as

$\left\{\begin{array}{l}\boldsymbol{m} \ddot{\boldsymbol{x}}_{1}+\boldsymbol{c} \dot{\boldsymbol{x}}_{1}+\boldsymbol{k} \boldsymbol{x}_{1}=\boldsymbol{T} \\ x=x_{n} \\ F_{\mathrm{A}}=m_{\mathrm{f}} \ddot{x}+c_{\mathrm{f}} \dot{x}+k_{\mathrm{f}} x\end{array}\right.$,

where the mass matrix

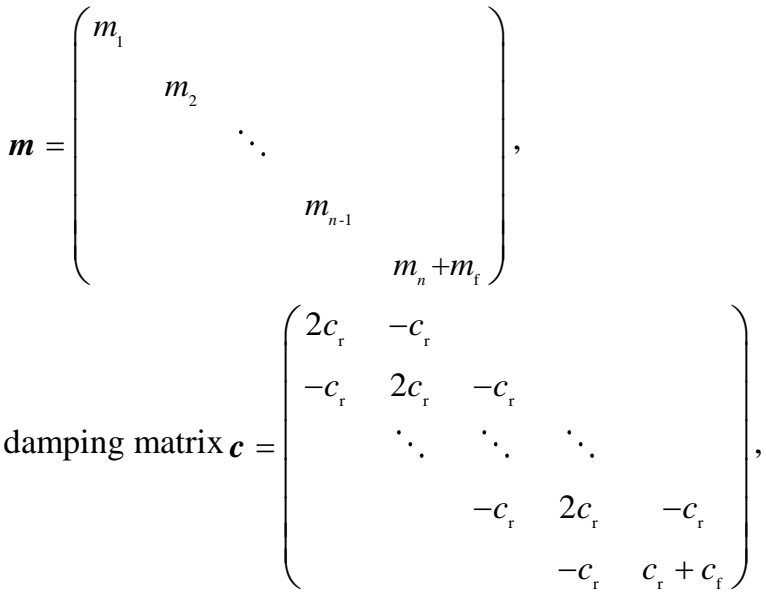

$$
\begin{aligned}
& \text { stiffness matrix } \boldsymbol{k}=\left(\begin{array}{ccccc}
2 k_{\mathrm{r}} & -k_{\mathrm{r}} & & & \\
-k_{\mathrm{r}} & 2 k_{\mathrm{r}} & -k_{\mathrm{r}} & & \\
& \ddots & \ddots & \ddots & \\
& & -k_{\mathrm{r}} & 2 k_{\mathrm{r}} & -k_{\mathrm{r}} \\
& & & -k_{\mathrm{r}} & k_{\mathrm{r}}+k_{\mathrm{f}}
\end{array}\right) \text {, }
\end{aligned}
$$

displacement vector $x_{1}=\left(\begin{array}{lllll}x_{1} & x_{2} & \ldots & x_{n-1} & x_{n}\end{array}\right)^{\mathrm{T}}$, driving force distribution on each element $T=\left(\begin{array}{lllll}q_{1} & q_{2} & \ldots & q_{n-1} & q_{n}\end{array}\right)^{\mathrm{T}}, x$ is the practical output displacement of the actuator and equal to the response of the $n^{\text {th }}$ element $x_{n}, F_{\mathrm{A}}$ denotes respectively the displacement and force of GMM bar. The initial condition is $x_{1}(0)=0, \dot{x}_{1}(0)=0$.

With respect to $q_{i}$, the equivalent magnetostrictive force will elongate in length of each element. Since it is the spring rather than mass that take up this assumed length, the above force is exerted on both ends of the spring. 
Exhibited as Fig. 3, $f_{i}$ is regarded as the average of total work within a certain element, take $L_{0}$ as the original length of GMM bar, then

$f_{i}=\frac{n}{L} \int_{L_{0}+\frac{i-1}{n} L}^{L_{0}+\frac{i}{n}} A E^{H} \lambda(I, x) \mathrm{d} x$.

The driving force of each element is

$q_{i}=f_{i}-f_{i+1} \quad(i=1,2, \cdots, n-1) \quad$ and $\quad q_{n}=f_{n}$.

The assumption could be verified by a simplified case. If the GMM bar is located in a unified field environment, the driving force in both ends of GMM bar is equal (only the upper end is loaded), since the assumed stiffness is constant, the GMM bar will elongate evenly, on the other hand, if the force is loaded directly on each element, the quantity of force will increase when the mesh is finer and the elongation will varies in elements, leading to a uneven deformation.

\subsection{Calculation of response}

Under a step excitement, the magnetic strength, magnetization, magnetostriction, force and displacement reach some constants. The stable performance will not be interfered by mass and damping, therefore the differential items equals zero. Remark $T_{\mathrm{O}}$ as the stable vector, the stable displacement vector $x_{10}$, stable displacement of GMA $x_{\mathrm{O}}$ and stable output force $F_{\mathrm{AO}}$ could be calculated as

$$
\begin{aligned}
x_{\mathrm{O}}=(0,0, \cdots, 1)_{1 \times n} \boldsymbol{x}_{1 \mathrm{O}}, F_{\mathrm{AO}} & =k_{2} x_{\mathrm{O}} \\
\text { where } & \boldsymbol{x}_{1 \mathrm{O}}=\boldsymbol{K}^{-1} \boldsymbol{T}_{\mathrm{O}}
\end{aligned}
$$

In classical analysis, the transient response is usually obtained by modal superposition. However, due to the damping of GMM rod is large and the non-diagonal elements in matrix $C$ are always too large to overlook. Under this condition, matrix in (9) are not so readily to be decoupled, which is elaborate to get a effective analytical solution via modal analysis. In this paper, the numerical method, based on MATLAB, is employed to calculate the transient response. The system is transformed in a state space form

$$
\left\{\begin{array}{l}
\dot{x}_{1}=x_{2} \\
\dot{x}_{2}=m^{-1}\left(T-k x_{1}-c x_{2}\right)
\end{array}\right. \text {. }
$$

Take $\boldsymbol{x}=\left(\begin{array}{ll}\boldsymbol{x}_{1} & \boldsymbol{x}_{2}\end{array}\right)^{\mathrm{T}}$, and the GMA system could be expressed as

$$
\left\{\begin{array}{l}
\dot{x}=\left(\begin{array}{cc}
0 & E \\
-m^{-1} k & -m^{-1} c
\end{array}\right) x+\left(\begin{array}{cc}
0 & 0 \\
0 & m^{-1}
\end{array}\right)\left(\begin{array}{l}
0 \\
T
\end{array}\right), \\
x_{l}=(0,0, \cdots, 1)_{1 \times n}\left(\begin{array}{ll}
E & 0
\end{array}\right)_{n \times 2 n} x
\end{array}\right.
$$

where $E$ denotes identity matrix. In zero initial condition, the output displacement of GMA is expressed as a vector. For the overall system, the response time denotes the period it takes when the last element reaches the stable status.

\subsection{Calculation of DOF}

Calculated results of steady-state displacement and responding time are shown in Fig. 4. From Fig. 4, computed results become stable with $n$ increasing, and if $n$ is selected too small, the computing error will be quite high. When $n=1$, a tremendous error of computed displacement reaches $1.8 \mu \mathrm{m}$ compared to the steady-state value, and the error of response time is about $6 \mu \mathrm{s}$. On the other hand, there is no obvious variation is observed in computing precision after $n=30$. As a result, $n$ is selected as 30 to balance the computing precision and velocity.
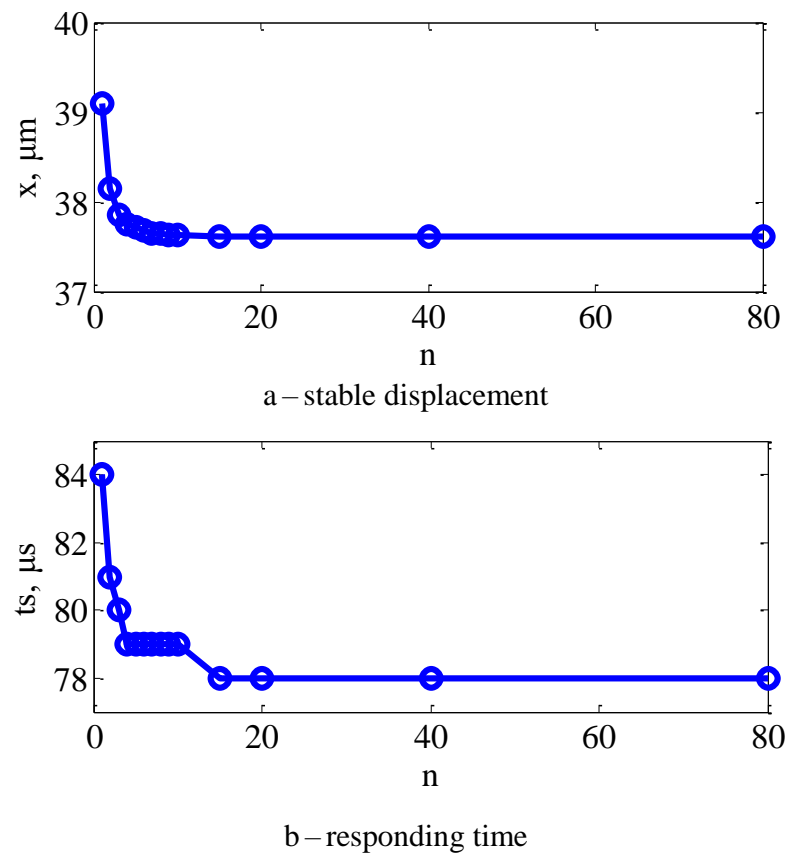

Fig. 4. Responses under different DOFs

\section{MODEL VALIDATION}

\subsection{Experimental setup}

Parameters of GMA are layout as Table. 1. The input signal is generated by signal generator PS2000 and enlarged by a particular power amplifier GF800. Then the displacement signal of GMA is acquired by displacement sensor HN808 and feedbacked to master computer.

Table 1. Parameters of the GMA

\begin{tabular}{|c|c|}
\hline Parameters & Value \\
\hline Length, $\mathrm{mm}$ & 39.5 \\
\hline Diameter, $\mathrm{mm}$ & 5 \\
\hline Elastic modulus, $\mathrm{N} / \mathrm{m}^{2}$ & $3 \times 10^{10}$ \\
\hline Non-hysteresis coefficient, $\mathrm{A} / \mathrm{m}$ & 7050 \\
\hline Magnetization parameter & -0.01 \\
\hline Saturation magnetization, $\mathrm{A} / \mathrm{m}$ & $8.0 \times 10^{5}$ \\
\hline Saturation strain & $0.8 \times 10^{-3}$ \\
\hline Reversible coefficient & 0.18 \\
\hline Inside diameter, $\mathrm{mm}$ & 15 \\
\hline Outside diameter, $\mathrm{mm}$ & 28 \\
\hline Coil turns & 1033 \\
\hline Stiffness, $\mathrm{N} / \mathrm{m}$ & $1.7 \times 10^{4}$ \\
\hline Length of the windings, $\mathrm{mm}$ & 40 \\
\hline Irreversible coefficient, $\mathrm{A} / \mathrm{m}$ & 3208 \\
\hline
\end{tabular}




\subsection{Discussion}

Model described in Eq. 1 and Eq. 3 are more appreciated in DC driving or low frequency cases, therefore, a DC current and $5 \mathrm{~Hz}$ sine wave is selected as driving signal. Under different DC driving levels, namely, $0,0.5,1.0,1.5,2.0,2.5,3.0,3.5,4.0,4.5,5.0 \mathrm{~A}$, the calculated and test results are compared as Fig. 5. As the single degree of freedom model is widely used in other references, the calculated result of single DOF model is also shown.

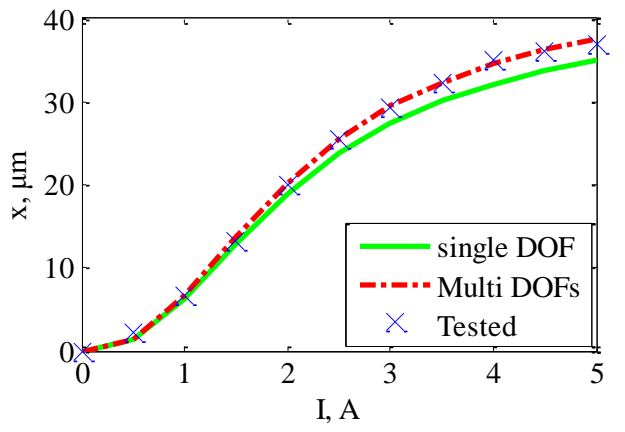

According to Fig. 5, the calculated results of multiple DOFs model matches with the test curves adequately, with the largest error beneath $1.0 \mu \mathrm{m}$ under $I=2.5 \mathrm{~A}$ and the relative error less than $4 \%$ approximately. Then proposed displacement model is verified. It can also be drawn that the multiple DOFs model is better than the traditional single DOF model in descrbing the actuator response.

Under $10 \mathrm{~Hz}$ and $5 \mathrm{~Hz}$ sine signals with different driving level, the calculated and test results are exhibited as Fig. 6. According to the test result, there is no obvious bias in 'double frequency' effect, amplitude, shape and saturation between test and calculated results. The comparison indicates there is no tremendous error accumulation in calculation process and the precision is satisfactory. Moreover, abutting $I=2.5 \mathrm{~A}$, the calculated result via proposed multi-DOF model and the classical single DOF model are $26.1 \mu \mathrm{m}$ and $27.9 \mu \mathrm{m}$ respectively. Test result under this condition is $25.2 \mu \mathrm{m}$, which means that in our particular case, the proposed algorithm reaches a higher precision in displacement computing.

Fig.5. Stable-state response with DC input
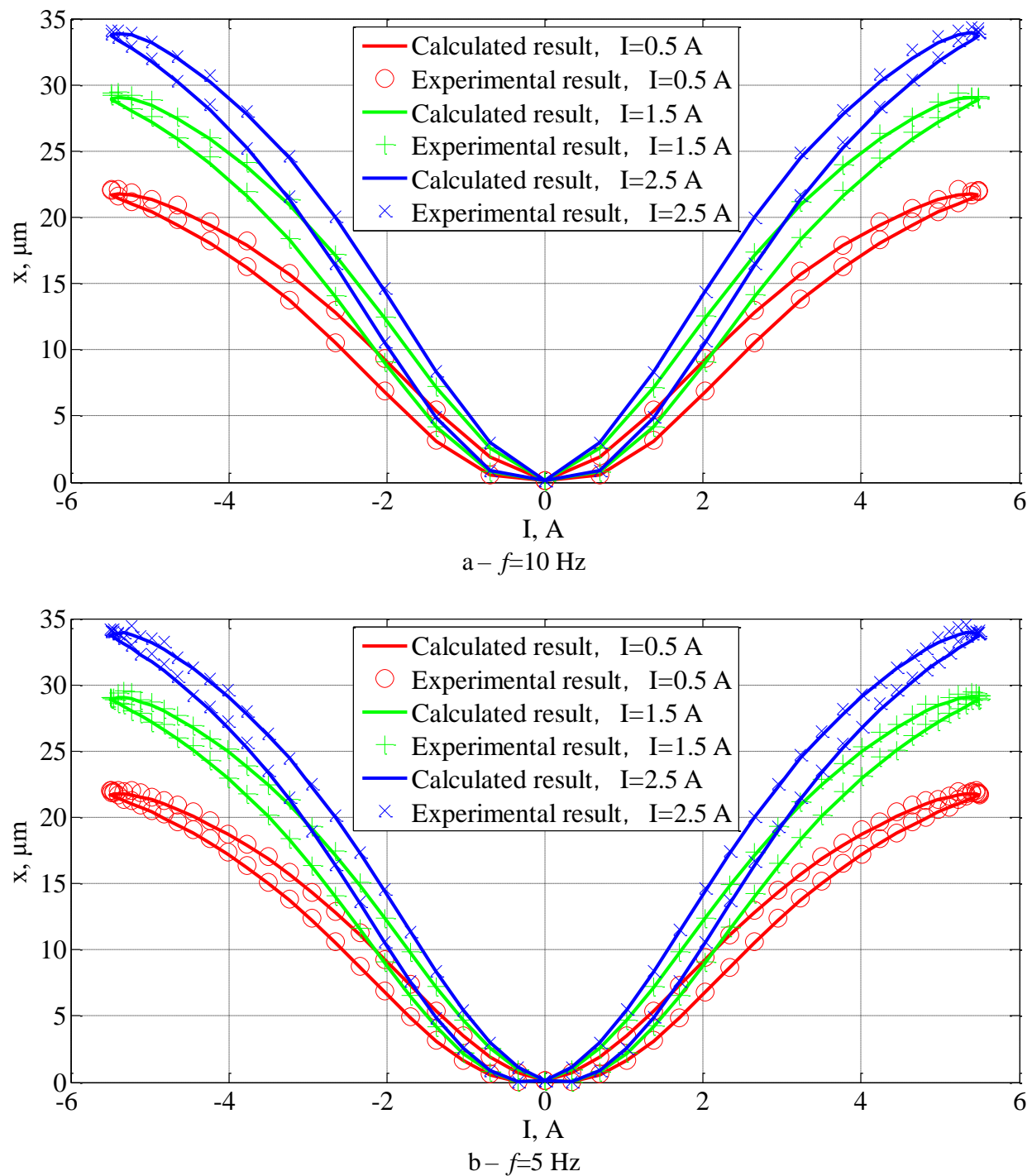

Fig. 6. Sinusoidal responses under different frequencies 


\section{CONCLUSIONS}

1. A specific GMA structure is developed to embed into a novel oil injector. The elongation of GMM rod is transformed as displacement of output shafted, and accompanied with the disk spring, the oil injection is controlled more precisely and swiftly.

2. A specific model is proposed with multi-DOFs to account for the uneven distribution of magnetic field along axial dimension. The quantity of DOFs is determined by analysis its interference on computing precision.

3. According to the comparison between calculated and test results, proposed multi-DOF model are more amendable to describe to output characteristics of GMA. The largest error is about $1.0 \mu \mathrm{m}$, and the relative error is beneath $4 \%$. Under low frequency drive, calculated result matches more satisfactorily with the test curve compared with the single DOF model.

\section{Acknowledgments}

This paper was supported by National Natural Science Foundation of China under Grant No. 51275525.

\section{REFERENCES}

1. Quandt, E., Seemann, K. Fabrication and Simulation of Magnetostrictive Thin-film Actuators Sensors and Actuators A: Physical 50 1995: pp. 105-109. https://doi.org/10.1016/0924-4247(96)80092-6

2. Claeyssen, F., Lhermet, N., Letty, R.L., Bouchilloux, P. Actuators, Transducers and Motors Based on Giant Magnetostrictive Materials Journal of Alloys and Compounds 258 (1) 1997: pp. 61-73. https://doi.org/10.1016/S0925-8388(97)00070-4

3. Park, Y.W., Kim, D.Y. Development of a Giant Magnetostrictive Microactuator Journal of Magnetism and Magnetic Materials 272 (5) 2004: pp. e1765-e1766. https://doi.org/10.1016/j.jmmm.2003.12.991

4. Zhang, J., Wang, H., Cao, S. Modeling of a Giant Magnetostrictive Device for Magnetic Force Control Based on Inverse Magnetostrictive Effect Journal of Mechanical Engineering 44 (5) 2008: pp. 51-56. https://doi.org/10.3901/JME.2008.05.051

5. Li, Y., Zhu, Y., Wu, H., Tian, Y., Niu, S. The Magnetic Field Modelling and Analysis of Giant Magnetostrictive
Actuator for Jet Servo Valve Journal of China Ordnance 31 (12) 2010: pp. 1587-1592.

6. Yang, B., Xu, P., Meng, G., Yang, D., Xu, W., Tan, X. Design and Control of Giant Magnetostrictive Actuator for Long-stroke Precision Positioning Journal of Mechanical Engineering 48 (1) 2012: pp. 25-31.

7. Lu, Q., Chen, D., Wei, G. Development of Giant Magnetostrictive Materials and Its Application in the Field of Precision Actuators Journal of Hubei University of Technology 21 (3) 2006: pp. $92-94$.

8. Yuan, F., Lin, X., Huang, Y., Gao, Y. Investigation on Effect of High Pressure Common Rail Injection System Parameters on Diesel Engine Performance Chinese Internal Combustion Engine Engineering 33 (2) 2012: pp. 11-18.

9. Hong, C.C. Application of a Magnetostrictive Actuator Materials and Design 46 2013: pp. 617-621. https://doi.org/10.1016/j.matdes.2012.11.013

10. Tanana, H., Sato, Y., Urai, T. Development of a Commonrail Proportional Injector Controlled by a Tandem Arrayed Giant Magnetostrictive Actuator JSAE Review 22 2001: pp. 369-371. https://doi.org/10.4271/2001-01-3182

11. Lv, F., Xiang, Z., Qi, Z., Cheng, Y. The Design of Highspeed Powerful Solenoid Based on Giant Magnetostrictive Material and Analysis of Its Control Method Transactions of CSICE 18 (2) 2000: pp. 199-202.

12. Braghin, F., Cinquemani, S., Resta, F. A Model of Magnetostrictive Actuators for Active Vibration Control Sensors and Actuators A: Physical 165 2011: pp. $342-350$. https://doi.org/10.1016/j.sna.2010.10.019

13. Zhao, G. MATLAB Practice and Control System Simulation Beijing: Beijing University of Aeronautics and Astronautics Press, 2012: pp. 53-65.

14. Benabou, A., Leite, J.V., Clenet, S., Siman, C., Sadowski, N. Minor Loops Modelling with a Modified JilesAtherton Model and Comparison with the Preisach Model Journal of Magnetism and Magnetic materials 320 2008: pp. e1034-e1038. https://doi.org/10.1016/j.jmmm.2008.04.092

15. Chwastek, K. Frequency Behaviour of the Modified JilesAtherton Model Physica B 403 2008: pp. 2484-2487. https://doi.org/10.1016/j.physb.2008.01.010

16. Szewczyk, R., Bienkowski, A., Salach, J. Extended JilesAtherton Model for Modelling the Magnetic Characteristics of Isotropic Materials Journal of Magnetism and Magnetic materials 320 2008: pp. e1049-e1052. https://doi.org/10.1016/j.jmmm.2008.04.107 